\title{
Chilblain-like lesions likely associated with coronavirus disease 2019: A Canadian case presentation
}

\author{
Robin Glicksman, $\mathrm{MSc}^{*}$; Catherine Varner, $\mathrm{MD}, \mathrm{MSc}^{\dagger \ddagger \S}$
}

Keywords: Acral ischemia, chilblains, coronavirus, COVID-19, cutaneous, pernio

\section{INTRODUCTION}

Coronavirus disease 2019 (COVID-19) emerged in Wuhan, China, in December 2019 and has since been declared a global pandemic. While initially thought to be a respiratory illness, it is becoming increasingly apparent how little is known about COVID-19 and its clinical manifestations. Illness from COVID-19 can range from asymptomatic to fatal with the most widely referenced symptoms being fever, dry cough, nasal congestion, fatigue, myalgia, asthenia, anosmia, and diarrhea. ${ }^{1}$ Recently, it has been proposed that COVID-19 could be associated with dermatological manifestations. ${ }^{1-5}$ We describe herein the case of a patient who presented to the emergency department (ED) with chilblain-like lesions apparently induced by COVID-19.

\section{CASE PRESENTATION}

A 30-year-old female presented to the ED with a 4-day history of acute-onset, burning, tender toe lesions. Three weeks before lesion onset, the patient experienced a 5-day course of fevers, cough, and diarrhea, after close contact with a confirmed COVID-19 case. The patient was unable to access telecare during her illness, did not seek in-person care, and was not tested for COVID-19.
Topical hydrocortisone cream applied to the lesions provided no symptomatic improvement. The patient's medical and surgical history was otherwise unremarkable, and she was not currently taking any medications. The patient did not drink alcohol but used marijuana recreationally. Prior to presenting, the patient was on home confinement for 5 weeks.

On presentation to ED triage, the patient's vital signs were stable and did not change in a clinically significant way while in the ED.

Physical examination was notable for several violaceous nodules bilaterally on the dorsal aspect of the toes with associated dusky discolouration of the toes. The lesions were tender to palpation (Figures 1 and 2). A complete skin examination was otherwise unremarkable.

A complete blood cell count; erythrocyte sedimentation rate; D-dimer test; low-density plasma; and biochemistry panel (random glucose, sodium, potassium, chloride, bicarbonate, anion gap, and creatinine) were all within normal limits. The consulting rheumatologist also suggested C-reactive protein, IgG immunoglobin, IgA immunoglobin, IgM immunoglobin, complements C3 and C4, and all were within normal limits. The patient's serology screening for hepatitis B core IgM antibody, infectious mononucleosis, and parvovirus B19 IgM was negative. Blood culture, anti-proteinase 3 antibody, and anti-myeloperoxidase antibody results were unremarkable. A urinalysis showed a +2 leukocyturia, trace hematuria, +1 proteinuria, and her protein to creatinine ratio was 5.4. Finally, a polymerase chain reaction nasopharyngeal swab for COVID-19 was negative.

From the ${ }^{*}$ Faculty of Medicine, University of Toronto, Toronto, ON; ${ }^{\dagger}$ Department of Family and Community Medicine, University of Toronto, Toronto, ON; ${ }^{\ddagger}$ Schwartz/Reisman Emergency Medicine Institute, Toronto, ON; and ${ }^{\S}$ Mount Sinai Hospital, Toronto, ON.

Correspondence to: Dr. Catherine Varner, Sinai Health System, Mount Sinai Hospital, 206-600 University Avenue, Toronto, ON M5G 1X5; Email: catherine.varner@sinaihealth.ca

(c) Canadian Association of Emergency Physicians 2020

CJEM 2020;22(5):611-613

DOI 10.1017/cem.2020.423 


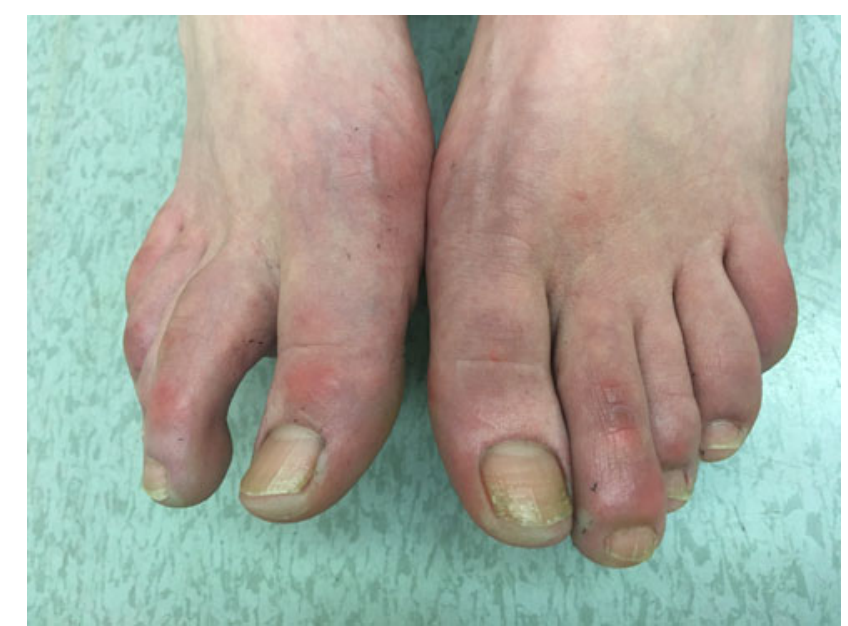

Figure 1. Photograph of Chilblain-like lesions on the toes of a 30-year-old female in the emergency department.

The rheumatologist agreed, in the context of recent flu-like illness, that the lesions were likely COVID-19-induced and were not associated with any systemic manifestations. The patient was discharged from the ED with a follow-up appointment at a virtual rheumatology clinic in 2 weeks. She was advised to return to the ED if she developed any worsening symptoms.

\section{DISCUSSION}

Chilblain inflammation is an abnormal reaction to cold and damp environments, characterized by painful cutaneous lesions involving the distal extremities. Rarely, chilblains are associated with systemic disease like systemic lupus erythematosus. This patient presented to the ED with no underlying risk factors for lesion development. It is particularly unusual for chilblain lesions to appear in warmer spring temperatures, especially in the

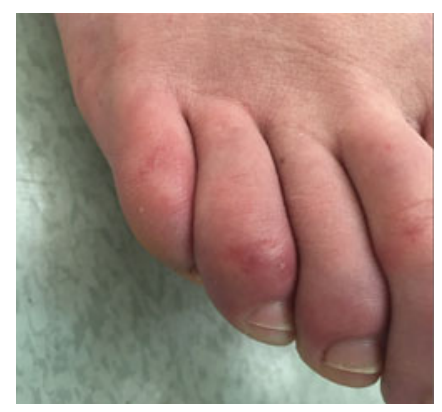

Figure 2. Photograph of Chilblain-like lesions on the toes of a 30 -year-old female in the emergency department. context of home confinement. ${ }^{2}$ Investigations of this patient also did not reveal any systemic disease. In this context, and based on observations reported elsewhere, it is suspected that these lesions were induced by COVID-19.

In Europe, case reports and case series have described COVID-19-associated skin lesions, highlighting an outburst of chilblain-like lesions contemporaneously related to the COVID-19 epidemic., ${ }^{2,3}$ Chilblain-like lesions were first reported by Mazzotta et al. ${ }^{6}$ in a case study of a child with suspected COVID-19. Since then, nationwide studies have explored the association between COVID-19 and cutaneous symptoms. In Spain, a "pseudo-chilblain" clinical pattern was identified in a prospective study of 375 patients presenting with cutaneous findings and confirmed COVID-19. ${ }^{4}$ Similar studies have since been published in France and Italy supporting these clinical findings. ${ }^{1,5}$

Our patient's clinical presentation is nearly identical to that reported in the medical literature. Chilblain-like lesions associated with COVID-19 tend to occur in pediatric populations who are often otherwise asymptomatic or young adults, like our patient, who tend to present with flu-like symptoms 3-4 weeks before lesion onset. ${ }^{3}$ Like our patient, lesions are reported to appear as violaceous macules, which can sometimes be painful or itchy and typically target the feet, particularly the dorsal surface of the toes, though there have been some reports of digit involvement as well. ${ }^{4,5}$

Unfortunately, COVID-19 testing was not completed while this patient had illness symptoms. Though her symptoms might be consistent with other viral infections, given her known COVID-19 exposure and the recent association between similar lesions and confirmed cases, it is strongly suspected that this patient had been infected with COVID-19.

Interestingly, our patient tested negative for COVID-19 virology in the ED. This is consistent with published literature, which suggests that some patients have no demonstrable evidence of active infection when presenting with such lesions., ${ }^{2,4}$ It has been hypothesized that these cutaneous findings could be a delayed manifestation of COVID-19. ${ }^{4}$ In European countries such as France, the outbreak of these lesions was only reported during the third week of containment, providing support to this hypothesis. ${ }^{3}$ To date, the exact mechanism of these lesions remains unknown; however, several hypotheses suggest that vasculopathy is involved in the pathogenesis. ${ }^{1}$ 
As the COVID-19 pandemic continues to evolve, there will likely be an increase in chilblain-like presentations to the ED. To date, these lesions have been reported to be self-limiting and resolve favourably. ${ }^{3}$ Unless there is evidence of further systemic vasculopathy, treatment in the ED should be supportive, aimed at symptom management and prevention of complications related to skin breakdown. It is uncertain whether medications used for chilblains to increase digital blood flow and decrease pain, such as calcium channel blockers, would be useful for this COVID-19 manifestation.

\section{CONCLUSION}

In summary, the toe lesions observed in this patient are suspected to be a manifestation of COVID-19. In the present pandemic, ED clinicians should be aware of this potential COVID-19 cutaneous manifestation to optimize management and contact tracing procedures.

Competing interests: None declared.

\section{REFERENCES}

1. De Masson A, Bouaziz J-D, Sulimovic L, et al. Chilblains are a common cutaneous finding during the COVID-19 pandemic: a retrospective nationwide study from France. 7 Am Acad Dermatol 2020; epub, doi:https://doi.org/10.1016/j.jaad.2020.04.161

2. Lopez-Robles J, de la Hera I, Pardo J, et al. Chilblain-like lesions: a case series of 41 patients during the COVID-19 pandemic. Clin Exp Dermatol 2020; epub, doi:https://doi.org/10. 1111/ced.14275

3. Landa N, Mendieta-Eckert M, Fonda-Pascual P, et al. Chilblainlike lesions on feet and hands during the COVID-19 pandemic. Int 7 Dermatol 2020; epub, doi:https://doi.org/10.1111/ijd. 14937

4. Galvan Casas C, Catala A, Carretero Hernandez G, et al. Classification of the cutaneous manifestations of COVID-19: a rapid prospective nationwide consensus study in Spain with 375 cases. Br 7 Dermatol 2020; epub, doi:https://doi.org/10. 1111/bjd.19163

5. Piccolo V, Neri I, Filippeschi C, et al. Chilblain-like lesions during COVID-19 epidemic: a preliminary study on 63 patients. 7 Eur Acad Dermatol Venereol 2020; epub, doi:https:// doi.org/10.1111/jdv.16526

6. Mazzotta F, Troccoli T. Acute acro-ischemia in the child at the time of COVID-19. Eur 7 Pediat Dermatol 2020; 30(2): 71-74. 